\title{
EVALUAR LA ALFABETIZACIÓN CIENTÍFICA EN EL PROGRAMA DE LA OECD PARA LA EVALUACIÓN INTERNACIONAL DE ESTUDIANTES (PISA) ${ }^{1}$
}

\author{
HARLEN, WYNNE \\ University of Bristol
}

Resumen. Este trabajo se refiere a qué se está evaluando y por qué, en el Programme for International Student Assessment (PISA) conducido por la OECD. El trabajo se ocupa de las razones para el programa y considera la definición de alfabetización científica que subyace a los materiales de evaluación, cómo se pone ésta en acción en el enfoque y los materiales de evaluación, y la forma en que se difundirán los resultados.

Palabras clave. Alfabetización científica, evaluación, programa internacional.

Summary. This presentation concerns what is being assessed, and why, in the OECD Programme for International Student Assessment (PISA). The paper deals with the reasons for the programme and considers the definition of scientific literacy which underpins the test materials, how it is played out in the test approach and the form in which the results are to be reported. Keywords. Scientific literacy, assessment, international programme.

\section{INTRODUCCIÓN}

Este trabajo se refiere a qué se está evaluando y por qué, en el Programa de la OECD para la Evaluación Internacional de Estudiantes (Programme for International Student Assessment, PISA). Los resultados de la primera ronda de encuestas a estudiantes estarán disponibles a fines de 2001, pero la concepción de alfabetización científica y los métodos usados para evaluarla son de interés en sí mismos, así como esenciales para la interpretación de los eventuales resultados.

Este trabajo se ocupa brevemente de las razones para el programa antes de considerar la definición de alfabetización científica que subyace a los materiales de evaluación, cómo se pone ésta en acción en el enfoque y los materiales de evaluación, y la forma en que se difundirán los resultados. Esto último hace explícita la manera en que se concibe el desarrollo en la alfabetización científica.

\section{FUNDAMENTOS DEL PISA}

El propósito del PISA es proveer indicadores de salida de algunos sistemas educativos nacionales con respecto a cuatro objetivos principales de la educación, descritos como la alfabetización en la lectura, en las matemáticas y en las ciencias, y la habilidad en la resolución de problemas. La OECD, desde 1988, ha estado financiando investigaciones para definir indicadores de educación que permitan hacer comparaciones válidas y ha venido reportando los resultados de su aplicación en Education at a Glance (publicada periódicamente desde 1991). Estos indicadores conciernen principalmente mediciones de entrada, tales como recursos, tiempo dedicado a las diferentes asignaturas, preparación de los profesores, costo por estudiante y mediciones de salida, tales como destino de los egresados escolares. El interés por comparar el rendimiento de los estudiantes sólo puede ser satisfecho usando datos de estudios internacionales, tales como las encuestas de IEA e IAEP, pero, por varias razones, estos datos no se ajustaban a la necesidad de los gobiernos, de acuerdo con la OECD, de tener información relevante, confiable y, sobre todo, regular, que pudiera ayudar en las decisiones de política educativa. La OECD, por tanto, estableció su propio programa, diseñado para satisfacer la necesidad de evaluación y para proveer indicadores relativos al rendimiento de los estudiantes de 15 años de edad, indicadores para «contribuir a la comprensión del grado en que los sistemas educativos de los países participantes están preparando a sus estudiantes para ser aprendices durante 
toda la vida y para jugar roles constructivos como ciudadanos en la sociedad» (OECD, 1999, p. 7).

Así, el PISA se estableció para evaluar en qué medida, al final de la educación obligatoria, los estudiantes han adquirido las habilidades y la comprensión, en los campos identificados, necesarias para que los jóvenes participen efectivamente en la sociedad. Aunque las ciencias y las matemáticas corresponden a asignaturas escolares, el PISA no trata de evaluar cuán bien los estudiantes dominan el contenido curricular, sino más bien qué habilidades generales y qué comprensión amplia han adquirido. Así, es importante distinguir entre evaluar la alfabetización científica y evaluar la «ciencia».

Las principales características del programa diseñado para alcanzar este objetivo son las siguientes:

- Una muestra de estudiantes de 15 años en cada país participante es examinada a intervalos de tres años, a partir de 2000.

- Cada evaluación incluye ítems de los tres campos de alfabetización.

- Todos los campos de alfabetización son examinados en cada encuesta; uno de ellos es el campo principal y los otros una parte menor de la encuesta. La alfabetización lectora es el campo principal en 2000, las matemáticas en 2003 y las ciencias son el campo principal en 2006. A partir de 2003 se incluirá también la resolución de problemas.

- Cada campo es definido en términos de conocimientos y habilidades importantes que se necesitan en la vida adulta. Los ítems de evaluación dan prioridad al dominio de procesos, la comprensión de conceptos y la capacidad para funcionar en situaciones variadas basadas en la vida real.

- Se recoge información contextual por medio de un cuestionario a los estudiantes y a los directores de las escuelas.

- Los resultados serán las tendencias en el tiempo, para cada país, del conocimiento y las habilidades conseguidos al final de la escolarización obligatoria, así como las comparaciones entre países y las relaciones entre los indicadores contextuales, las características de las escuelas y el rendimiento de los estudiantes.

- El programa no constituye un estudio longitudinal, aunque tal estudio, enfocado en la transición entre la escuela y el trabajo, está siendo considerado, junto con otros proyectos de investigación asociados.

En la primera ronda participaron treinta y dos países, veintiocho de los cuales son miembros de la OECD ${ }^{2}$. Desde entonces, otros doce países se han sumado al programa y están usando las pruebas de 2000 en 2001.

El proyecto es llevado a cabo, bajo la dirección general de la Secretaría de la OECD, por un consorcio liderado por el Australian Council for Educational Research
(ACER), el Instituto Nacional para la Medición Educacional en Holanda (CITO), el Educational Testing Service (ETS), el Instituto Nacional para la Investigación Educativa de Japón (NIER) y Westat en los Estados Unidos. El proyecto es dirigido por un Comité de Países Participantes, en el cual los países están representados a nivel político. El Comité tiene que consensuar todos los asuntos relacionados con las encuestas. Hay un Grupo de Asesoramiento Técnico que aconseja sobre todos los asuntos relacionados con la recolección y el análisis de datos, un panel de Revisión Cultural y, para cada campo, un Grupo de Expertos en la Asignatura (Subject Expert Group, SEG), cuyos miembros son elegidos, no como representantes de los países, sino por su experiencia individual en áreas relevantes. ${ }^{3}$

Éstos no son sólo detalles de gestión, puesto que es importante notar que lo que se evalúa es lo que los países participantes han acordado que son resultados deseables, estén éstos reflejados o no en los currículos actuales de cada país en particular. Eso constituye una diferencia con el TIMSS, que se dedicó a evaluar lo que es común en los currículos de los diferentes países. Otra diferencia entre TIMSS y PISA son los detalles de «cómo» el programa se lleva a cabo; esto se verá en la descripción del marco y las unidades de evaluación que se expone a continuación.

\section{EL MARCO DE EVALUACIÓN PARA LA ALFABETIZACIÓN CIENTÍFICA}

No es apropiado revisar aquí la variedad de formas en las que la alfabetización científica ha sido descrita y definida. Se consideraron varias descripciones y modelos existentes de alfabetización científica, muchos de los cuales han sido desde entonces revisados por Laugksch (2000). Como resultado de una considerable discusión, el grupo de expertos de ciencias desarrolló su propia definición de alfabetización científica:

La capacidad de usar conocimiento científico para identificar preguntas y para sacar conclusiones basadas en las pruebas, con el fin de entender y ayudar a tomar decisiones sobre el mundo natural y los cambios realizados en él a través de la actividad humana.

Con el beneficio de la revisión, la guía para esta formulación era más o menos la siguiente:

- En relación con los dos propósitos de la educación científica - producir futuros científicos y otorgar a todos los estudiantes una comprensión que mejore sus vidas futuras (Fensham, 1985)-, no cabía duda de que el PISA se ocupaba de este último.

- A pesar de que la alfabetización científica es algo a lo que se aspira y que se desarrolla durante la vida, es esencial que se comience en la escuela. 
- La alfabetización científica no debe ser identificada con el vocabulario; el término alfabetización fue interpretado metafóricamente para significar una competencia general o «estar cómodo» con las formas científicas de entender las cosas.

- Un rasgo central fue el uso de las pruebas (evidence); esto incluye el conocimiento de cómo se obtienen pruebas en las ciencias, qué hace que unas sean más importantes que otras, cuáles son sus insuficiencias y dónde pueden y deben ser aplicadas.

Debemos enfatizar que ésta es la terminología que se está usando actualmente. Puede ser modificada, ya que el proceso de operacionalización del concepto de alfabetización científica y los intentos de informar sobre ella fuerzan una constante reflexión y revisión.

Una afirmación tan amplia debe ser descompuesta a fin de ver lo que realmente significa en la práctica. El primer paso consistió en identificar sus dimensiones generales. Se consideró que éstas eran:

a) los procesos mentales involucrados en el tratamiento de una pregunta o un asunto;

b) el conocimiento científico y la comprensión conceptual requeridos al usar dichos procesos;

c) las áreas de aplicación de los procesos y los conceptos; d) la situación o el contexto de aplicación.

El segundo paso fue decidir los componentes relevantes de estas dimensiones, teniendo en cuenta la intención general de enfocarnos en la alfabetización más que en el dominio del contenido curricular. Las decisiones sobre estos componentes se revisarán brevemente ahora para cada dimensión. Se pueden encontrar más detalles en OECD $(1999,2000)$ y Harlen (2000).

\section{La dimensión de procesos}

En esta dimensión se han definido cuatro procesos:

1) Comprender los conceptos científicos, lo que significa la habilidad de hacer uso del conocimiento científico y mostrar comprensión de los conceptos de ciencias aplicando ideas científicas, información o conceptos apropiados, no dados, a una situación presentada. Esto puede involucrar explicar relaciones, eventos o fenómenos científicos o posibles causas de cambios. También puede involucrar hacer predicciones sobre los efectos de unos dados cambios o identificar los factores que influencian un determinado resultado.

2) Comprender la naturaleza de la investigación científica, lo que significa la habilidad para reconocer preguntas que pueden ser científicamente investigadas y para darse cuenta de qué implican estas investigaciones. También puede involucrar reconocer preguntas que podrían ser o bien están siendo contestadas en una investigación; o distinguir las preguntas que pueden ser resuel- tas por la investigación científica de las que no; o, de forma más abierta, sugerir una pregunta que podría ser investigada científicamente en una situación dada. Se incluye también identificar pruebas/datos necesarios para contrastar una explicación o explorar un asunto, lo que requiere, por ejemplo, identificar o reconocer qué cosas deben ser comparadas, qué variables deberían ser variadas o controladas, qué información adicional se necesita o qué acciones deben emprenderse para recoger datos relevantes.

3) Usar la evidencia científica, lo que significa la habilidad para dar sentido a los datos científicos como pruebas para afirmaciones o conclusiones. Esto puede involucrar sacar una conclusión a partir de pruebas/ datos científicos dados, o seleccionar de entre varias la conclusión que se ajusta a los datos. También puede involucrar dar razones a favor o en contra de una determinada conclusión en términos de los datos provistos o identificar las suposiciones hechas para llegar a una conclusión.

4) Comunicar descripciones o argumentaciones científicas, lo que significa la habilidad para comunicar, a otros, descripciones, argumentaciones y explicaciones científicas. Esto involucra comunicar a una audiencia específica conclusiones válidas a partir de las pruebas/ datos disponibles. También puede implicar la producción de una argumentación o una explicación basadas en la situación y en los datos dados o bien en información adicional relevante, expresada de una manera apropiada y clara para una determinada audiencia.

Enfatizamos que el uso de todos estos procesos requiere algunos conocimientos científicos. Esto resulta más obvio en el primer proceso, pero se aplica a los otros igualmente, ya que éstos no pueden ser descritos como «procesos científicos» a menos que se apliquen en relación con contenidos científicos.

\section{La dimensión de conceptos}

La selección y la expresión de conceptos científicos fue determinada a través de la consideración de las grandes ideas integradoras que ayudan a explicar aspectos de nuestro entorno material. No se hizo ningún intento de identificar todos los conceptos que satisfacen este criterio (evaluarlos todos en profundidad sería imposible dentro de los límites de espacio de las pruebas). En su lugar, los conceptos fueron muestreados a partir de los siguientes temas principales: estructura y propiedades de la materia, cambio atmosférico, cambios químicos y físicos, transformaciones de la energía, fuerzas y movimiento, forma y función, biología humana, cambio fisiológico, biodiversidad, control genético, ecosistemas, la Tierra y su lugar en el universo, cambio geológico.

\section{Las áreas de aplicación}

Los estudiantes que han adquirido alguna medida de alfabetización científica serán capaces de aplicar lo que 
han aprendido en la escuela a situaciones no escolares. La consideración de las áreas de aplicación de las ciencias que los ciudadanos del siglo XXI necesitarán entender y sobre las cuales habrán de tomar decisiones llevó a la identificación de 13 apartados, agrupados a grandes rasgos como pertenecientes a «la ciencia en la vida y la salud», «la ciencia en la Tierra y el medio ambiente», «la ciencia en la tecnología» (Fig. 1).

\section{Situaciones o contextos}

Los problemas relacionados con estas áreas de aplicación pueden afectarnos como individuos, como miembros de una comunidad local o como ciudadanos del mundo; a veces de las tres formas (p.e., en el caso de la contaminación). Además, algunas áreas de aplicación (p.e., salud, enfermedad y nutrición) tienen una historia que provee oportunidades para reconocer los cambios que ha experimentado la comprensión científica a lo largo de los años. Así, las situaciones pueden tener una relevancia personal, comunitaria, global o histórica. Las áreas de aplicación y las situaciones se combinan como muestra la figura 1 .

Figura 1

\begin{tabular}{|c|c|c|c|}
\hline Situación & \multicolumn{3}{|c|}{ Áreas de aplicación } \\
\hline & $\begin{array}{l}\text { La ciencia } \\
\text { en la vida } \\
\text { y la salud }\end{array}$ & $\begin{array}{l}\text { La ciencia } \\
\text { en la Tierra } \\
\text { y el medio } \\
\text { ambiente }\end{array}$ & $\begin{array}{l}\text { La ciencia } \\
\text { en la tecnología }\end{array}$ \\
\hline $\begin{array}{l}\text { Personal } \\
\text { Comunitaria } \\
\text { Global } \\
\text { Histórica }\end{array}$ & $\begin{array}{l}\text { Salud, } \\
\text { enfermedad } \\
\text { y nutrición. } \\
\text { Mantenimiento } \\
\text { y uso sostenible } \\
\text { de las especies. } \\
\text { Interdependencia } \\
\text { de los sistemas } \\
\text { físicos y } \\
\text { biológicos. }\end{array}$ & $\begin{array}{l}\text { Contaminación. } \\
\text { Producción } \\
\text { y pérdida } \\
\text { del suelo. } \\
\text { Meteorología } \\
\text { y clima. }\end{array}$ & $\begin{array}{l}\text { Biotecnología. } \\
\text { Uso de } \\
\text { materiales } \\
\text { y tratamiento } \\
\text { de residuos. } \\
\text { Uso de la } \\
\text { energía. } \\
\text { Transporte. }\end{array}$ \\
\hline
\end{tabular}

\section{UNIDADES DE EVALUACIÓN PARA LA ALFABETIZACIÓN CIENTÍFICA}

El formato de prueba para la alfabetización científica es una serie de unidades de prueba, cada una dedicada a un problema o asunto particular. Las unidades de prueba presentan a los estudiantes una situación de la vida real, tomada de una fuente auténtica y una serie de preguntas (ítems) sobre ella. Cada ítem requiere el uso de una de las habilidades de proceso y de algún conocimiento científico. La presentación del material de estímulo (problema o cuestión) requiere leer un texto, tabla o diagrama. Sin embargo, dado que varios ítems están conectados con el mismo material de estímulo en cada unidad, el tiempo total usado para leer en lugar de contestar las preguntas no es mayor que en una serie de ítems individuales de una prueba convencional. La dependencia de la lectura y la escritura es inevitable en una prueba escrita, que es el formato que asume actualmente la evaluación. El grupo de ciencias espera que en 2006, cuando las ciencias sean el componente principal, sea posible usar, como suplemento o como alternativa, instrumentos de evaluación implementados por ordenador.

Alrededor de dos tercios de los ítems usados en las encuestas estarán en una forma que pueda ser identificada como correcta o incorrecta sin ambigüedades. Estos ítems tienen una forma de respuesta fija o requieren dar sólo unas pocas palabras; son esencialmente ítems «cerrados». Otros ítems, «abiertos», requerirán respuestas extensas y podrán ser evaluados como parcialmente correctos, además de correctos o incorrectos. Pruebas piloto mostraron que el tiempo promedio para responder a un ítem era similar para los ítems abiertos y cerrados. Los criterios de evaluación de las preguntas abiertas proveen no sólo consignas generales sino también ejemplos de respuestas en cada categoría. Además, dado que estas respuestas dan información valiosa sobre las ideas y el pensamiento de los estudiantes, que luego puede ser usada en la planeación curricular, se usó un sistema de corrección de dos dígitos, a fin de poder guardar registro de las frecuencias de varios tipos de respuestas correctas e incorrectas (Angell et al., 2000).

Veintisiete unidades de evaluación de ciencias y unos pocos ítems sueltos constituyendo un total de 121 ítems, pasaron una prueba piloto en 1999. Después de considerar los datos estadísticos, los comentarios de los coordinadores de los programas nacionales, del Panel de Revisión Cultural y de los correctores, el número se redujo inicialmente a alrededor de 60 ítems. De éstos, el SEG de ciencias seleccionó los 35 ítems (doce unidades y 1 ítem suelto) que se incluirían en la primera encuesta. Estos ítems fueron elegidos de manera que abarcasen igualmente las tres áreas de aplicación y para obtener una distribución con respecto a los procesos dentro de los siguientes límites:

$\begin{array}{ll}\text { Proceso } 1 & 10-15 \% \\ \text { Proceso } 2 & 15-20 \% \\ \text { Proceso } 3 & 15-20 \% \\ \text { Proceso } 4 & 10-15 \% \\ \text { Proceso } 5 & 40-50 \%\end{array}$

Ejemplos de ítems de prueba

En la página web del PISA aparecen algunos ejemplos de ítems en unidades de prueba, junto con sus criterios de evaluación ${ }^{4}$. Los siguientes ejemplos, acompañados de breves comentarios ilustran ítems de dos unidades que pasaron la prueba piloto pero que no están siendo usadas en las encuestas.

- El ejemplo 1 es de una unidad llamada «Cambio climático».

Lee la siguiente información y contesta las preguntas que siguen. 


\section{¿Qué actividades humanas contribuyen al cambio climático?}

La quema de carbón, petróleo y gas natural, así como la deforestación y varias prácticas agrícolas e industriales, están alterando la composición de la atmósfera y contribuyendo al cambio climático. Estas actividades humanas han aumentado la concentración de partículas y de gases de efecto invernadero en la atmósfera.

La importancia relativa de las principales contribuciones al cambio en la temperatura se muestra en la figura 2.

\section{Figura 2}

Importancia relativa de las principales contribuciones al cambio en la temperatura de la atmósfera.

\begin{tabular}{|r|c|}
\hline Enfriamiento Importancia relativa Calentamiento \\
\hline Dióxido de carbono & \\
Metano & \\
& Partículas \\
& $\begin{array}{l}\text { Efectos de las partículas } \\
\text { en las nubes }\end{array}$ \\
\hline
\end{tabular}

Efectos conocidos

Efectos posibles

Fuente: adaptado de http://www.gcrio.org/ipcc/qa/04.html

Las barras que se extienden hacia la derecha de la línea central indican un efecto de calentamiento. Las barras que se extienden a la izquierda de la línea central indican un efecto de enfriamiento. El efecto relativo de las «partículas» y de los «efectos de las partículas sobre las nubes» es bastante incierto: en cada caso, el posible efecto se ubica en la zona mostrada por la barra gris.

La figura 2 muestra que el aumento de la concentración de dióxido de carbono y metano tiene un efecto de calentamiento. Las concentraciones de partículas causan un enfriamiento en dos formas, llamadas partículas y efectos de las partículas sobre las nubes.

Cambio climático: Ítem 1

Usa la información de la figura 2 para dar apoyo a la visión de que se debería dar prioridad a la reducción de la emisión de dióxido de carbono en las actividades humanas mencionadas.

\section{Comentario a la actividad}

El material estímulo para esta unidad está tomado de información sobre la contribución de varios factores al cambio climático encontrada en internet. Ésta es una fuente de información que los estudiantes actuales usa- rán cada vez más frecuentemente en su vida diaria. Así, resulta importante que ellos sean capaces de interpretar y usar dicha información. Sin embargo, se ha añadido una breve explicación para que la forma de presentación de los datos no represente un obstáculo en el uso de los datos para resolver la tarea propuesta. Éste es un tópico en el cual la evidencia no es concluyente en relación con las posibles decisiones a tomar.

El ítem 1 requiere que los estudiantes usen la información que se les da para dar apoyo a un curso de acción particular, que es reducir la emisión de dióxido de carbono como resultado de la actividad humana. Otro ítem en la unidad (no incluido aquí) pide a los estudiantes que usen la misma información de manera diferente, para apoyar la visión de que los efectos de la actividad humana no constituyen un problema real. En ambos casos, los ítems están evaluando la capacidad de comunicar conclusiones basadas en pruebas. Combinadas, las dos preguntas ejemplifican la cautela con la cual se debe aplicar la información científica en casos tan complejos. Responder a los ítems depende del conocimiento de contenidos tales como por qué el calentamiento y el enfriamiento son importantes en el cambio climático y cómo el dióxido de carbono, el metano y las partículas en el aire pueden producir estos efectos. Sin embargo, el énfasis está puesto en la construcción de una argumentación usando los datos obtenidos; por tanto, lo que se evalúa es la capacidad de comunicar eficazmente. Se valoran las respuestas que identifican la información relevante para la argumentación que se está haciendo. Este ítem se relaciona con el marco teórico de la siguiente manera:

- Procesos que se evalúan: Proceso 4: Comunicar descripciones o argumentaciones científicas.

- Tema conceptual involucrado: El cambio atmosférico. - Área de aplicación: La ciencia en la Tierra y el medio ambiente (meteorología y clima).

- El ejemplo 2 es de la unidad llamada «Chocolate», que tiene tres ítems.

Lee más abajo el resumen de un artículo que apareció en el diario Daily Mail el 30 de marzo de 1998.

Un artículo de periódico cuenta la historia de una estudiante de 22 años, llamada Jessica, que sigue una «dieta del chocolate». Ella afirma permanecer sana, y con un peso estable de $50 \mathrm{k}$, comiendo 90 barras de chocolate por semana y prescindiendo de todos los demás alimentos con excepción de una comida completa cada cinco días. Un experto en nutrición comentó: «Me sorprende que alguien pueda vivir con una dieta así. Las grasas le dan a Jessica energía para vivir, pero no obtiene una dieta equilibrada. Hay algunos minerales y nutrientes en el chocolate, pero no suficientes vitaminas. Esta dieta podría generarle más adelante serios problemas de salud.»

\section{Chocolate: Ítem 1}

Un libro con valores nutricionales provee los datos sobre el chocolate que se muestran en la tabla I. Supón que todos estos datos pueden aplicarse al tipo de chocolate que Jessica está comiendo. Supón también que las barras de chocolate que ella come pesan 100 gramos cada una. 
Tabla I

Contenido nutricional de $100 \mathrm{~g}$ de chocolate.

\begin{tabular}{|c|c|c|c|c|c|c|c|c|}
\hline \multirow{2}{*}{ Proteinas (g) } & Grasas (g) & Carbohidratos (g) & \multicolumn{2}{|c|}{ Minerales } & \multicolumn{3}{|c|}{ Vitaminas } & Energía total (kJ) \\
\hline & & & Calcio (mg) & Hierro (mg) & A & B (mg) & C & \\
\hline 5 & 32 & 51 & 50 & 4 & - & 0,20 & - & 2.142 \\
\hline
\end{tabular}

De acuerdo con la tabla, $100 \mathrm{~g}$ de chocolate contienen 32 g de grasas y proporcionan 2142 kJ de energía. El nutricionista decía: «Las grasas dan a Jessica energía para vivir...».

Cuando se comen $100 \mathrm{~g}$ de chocolate, ¿toda la energía (2142 $\mathrm{kJ}$ ) viene de los $32 \mathrm{~g}$ de grasas? Explica tu respuesta usando datos de la tabla I.

\section{Comentario al ítem 1}

Esta unidad contiene varios ítems relacionados con la comprensión de una dieta sana, y el conocimiento de los diferentes tipos de alimentos que se necesitan para ella. Los ítems llevan a la conclusión de que la dieta del chocolate que sigue esta estudiante está lejos de ser saludable y, además, le proporciona más energía de la que necesita. Sin embargo, el hecho de que esta historia estímulo podría, por sí misma, inducir a los estudiantes a intentar algo similar llevó a excluir esta unidad de las encuestas. La presentamos aquí para ejemplificar un tópico motivador e importante y la variedad de ítems que se evalúan.

El ítem 1 presenta información sobre el valor nutricional del chocolate, dando por supuesto que se puede aplicar al tipo de chocolate consumido por Jessica. Se indica que el chocolate contiene algunos minerales y vitaminas, además de grasas, proteínas y carbohidratos. La pregunta concierne a la contribución energética de estos componentes y requiere el conocimiento de que la energía proviene de las grasas, las proteínas y los carbohidratos y no de los minerales y vitaminas, que tienen otra función en una dieta sana. Este conocimiento debe ser aplicado en la situación presentada; así, el ítem requiere no sólo recordar sino también aplicar el conocimiento en una situación de la vida real. El ítem 1 del chocolate se relaciona con el marco teórico de la siguiente manera:

- Procesos que se evalúan: Proceso 5: Comprender conceptos científicos.

- Tema conceptual involucrado: Transformaciones de la energía.

- Área de aplicación: La ciencia en la vida y la salud (salud, enfermedad y nutrición).

\section{Chocolate: Ítem 2}

La tabla II, muestra la cantidad diaria recomendada de nutrientes y de kilojoules para una mujer de actividad normal.

El artículo dice que la dieta de Jessica consiste en 90 barras de chocolate por semana, pero que ella mantiene su peso estable. Supón que las barras de chocolate que ella come pesan 100 gramos cada una. De los datos de las tablas I y II puedes calcular que es difícil creer que Jessica mantenga su peso con esta dieta.

Muestra esto por medio de un cálculo.

\begin{tabular}{|c|c|c|c|c|c|c|c|c|c|c|c|}
\hline \multirow[t]{2}{*}{ Fila } & \multirow{2}{*}{$\underset{(\text { años })}{\text { Edad }}$} & \multirow{2}{*}{$\begin{array}{r}\text { Peso } \\
(\mathrm{kg})\end{array}$} & \multirow{2}{*}{$\begin{array}{l}\text { Proteínas } \\
\quad(g)\end{array}$} & \multirow{2}{*}{$\begin{array}{c}\text { Grasas } \\
(g)\end{array}$} & \multirow{2}{*}{$\begin{array}{c}\text { Carbohi- } \\
\text { dratos } \\
(g)\end{array}$} & \multicolumn{2}{|c|}{ Minerales } & \multicolumn{3}{|c|}{ Vitaminas } & \multirow{2}{*}{$\begin{array}{c}\begin{array}{c}\text { Energía } \\
\text { total }\end{array} \\
\\
(k J) \\
(m g)\end{array}$} \\
\hline & & & & & & Calcio & $\begin{array}{c}\text { Hierro } \\
\text { (mg) }\end{array}$ & $\underset{(m g)}{A}$ & $\underset{(m g)}{\mathbf{B}}$ & $\underset{(m g)}{\mathrm{C}}$ & \\
\hline 1 & $16-19$ & 50 & 60 & 85 & 320 & 0,9 & 15 & 0,45 & 2,5 & 50 & 9180 \\
\hline 2 & 16-19 & 60 & 60 & 90 & 350 & 1,0 & 15 & 0,45 & 2,5 & 75 & 9660 \\
\hline 3 & $20-35$ & 50 & 60 & 80 & 300 & 0,7 & 12 & 0,45 & 2,3 & 40 & 8990 \\
\hline 4 & $20-35$ & 60 & 60 & 85 & 330 & 0,8 & 12 & 0,45 & 2,4 & 50 & 9240 \\
\hline
\end{tabular}




\section{Comentario al ítem 2}

El ítem 2 del chocolate continúa explorando la dieta del chocolate, proveyendo más información, en la tabla II, sobre valores energéticos. Esta parte de la unidad permite evaluar la capacidad de relacionar la pregunta con la información dada en dicha tabla II, un aspecto de la alfabetización lectora que es evaluado mediante una pregunta que aquí no aparece. El peso del ítem 2 que mostramos aquí está puesto en la identificación de las pruebas requeridas para verificar la afirmación de la estudiante de que su peso se mantiene estable. Aunque se necesitan algunos cálculos, éstos se minimizan al elegir los datos más relevantes necesarios para comparar la ingesta diaria real con la recomendada, ya sea en gramos o en kilojoules. Una pregunta adicional en la unidad pide posibles razones por las cuales Jessica podría lograr mantener su peso estable a pesar de su ingesta excesiva de energía. El ítem 2 evalúa los siguientes aspectos del marco teórico:

- Procesos que se evalúan: Proceso 2: Comprender la naturaleza de la investigación científica.

- Tema conceptual involucrado: Transformaciones de la energía.

- Área de aplicación: La ciencia en la vida y la salud (salud, enfermedad y nutrición).

\section{Chocolate: Ítem 3}

Los expertos en nutrición dijeron que Jessica «no está tomando suficientes vitaminas». Una de las vitaminas que falta en el chocolate es la vitamina C. Quizás ella compensa la falta de vitamina $\mathrm{C}$ incluyendo un alimento que contiene un alto porcentaje de vitamina $\mathrm{C}$ en su «comida completa cada cinco días».

La siguiente es una lista de tipos de alimento:

a) pescado;

b) fruta;

c) arroz;

d) verduras.

¿Qué dos tipos de alimentos de esta lista recomendarías a Jessica para darle la oportunidad de compensar su carencia de vitamina $\mathrm{C}$ ?
A 1 y 2
D 2 y 3
B 1 y 3
C 1 y 4

E $\quad 2$ y 4
F 3 y 4

\section{Comentario al ítem 3}

Esta unidad involucra la consideración de una dieta saludable y, en un ítem no incluido aquí, las consecuencias de no seguirla. En este ítem, una respuesta correcta depende del conocimiento de las fuentes de uno de los componentes más importantes de la dieta sana. Este conocimiento es necesario para que los estudiantes tomen decisiones fundamentadas sobre su propia dieta y la de los demás. Lo que el ítem evalúa se relaciona con el marco teórico de la siguiente manera:

- Procesos que se evalúan: Comprender los conceptos científicos.
- Tema conceptual involucrado: El cambio fisiológico.

- Área de aplicación: La ciencia en la vida y la salud (salud, enfermedad y nutrición).

\section{INFORMAR SOBRE LA ALFABETIZACIÓN CIENTIFICA}

La intención del proyecto PISA es dar a conocer los resultados en todos los campos en términos de escalas de suficiencia, de modo que el rendimiento pueda ser interpretado en relación con el tipo de ítems, y no sólo la cantidad, que son contestados correctamente. En la actualidad se están desarrollando cinco escalas. En la primera ronda de pruebas, dado que la ciencia tenía un papel menor y sólo se le asignaban 60 minutos del tiempo total, la base empírica para desarrollar escalas no es muy sólida. Sin embargo, a través de la combinación del trabajo teórico y de los datos empíricos, el SEG de ciencias ha comenzado a establecer una escala general para la alfabetización científica. La escala está basada en la dimensión de procesos; las otras dimensiones se usan para etiquetar los ítems y asegurarse de que están distribuidos uniformemente a través de las áreas principales de conceptos, aplicación y situación. El punto de partida para el desarrollo de la escala general fue considerar los ítems para cada uno de los cuatro procesos y desarrollar una escala para cada uno. La dificultad de los ítems fue usada como guía, pero no era determinante de dónde caen los ítems en la escala. Esto es así porque hay muchos factores que afectan la dificultad de cada ítem, así como los procesos y la demanda conceptual que el ítem pretende medir. Estos factores inevitables que están interfiriendo incluyen el formato, el enunciado y la carga de lectura del ítem, y la familiaridad de los estudiantes con el contexto, o su exposición reciente a las ideas que se están evaluando. A pesar de que se hicieron todos los esfuerzos posibles para reducir estas interferencias, éstas no pudieron ser completamente evitadas.

La escala general para la alfabetización científica está compuesta de subescalas basadas en la dimensión del proceso. Por ejemplo, para comprender los conceptos científicos, la escala que describe lo que el estudiante necesita ser capaz de hacer para completar una tarea en cada nivel es:

5) crear o usar modelos conceptuales sencillos para hacer predicciones o dar explicaciones;

4) usar conceptos científicos elaborados para hacer predicciones o dar explicaciones;

3) usar conceptos científicos para hacer predicciones o dar explicaciones;

2) usar conocimiento común de la ciencia para hacer predicciones o dar explicaciones;

1) recordar simple conocimiento científico factual (por ejemplo, nombres, reglas simples).

Para comprender la naturaleza de la investigación científica: 
5) analizar investigaciones científicas en relación con, por ejemplo, el diseño experimental, la identificación de lo que se está poniendo a prueba;

4) formular o identificar información adicional requerida en una investigación científica, de modo de poder sacar conclusiones válidas;

3) reconocer preguntas que pueden ser respondidas a través de la investigación científica o detalles de qué implica una investigación científica;

2) reconocer las variables que se deben controlar o las preguntas que se pueden responder en contextos simplificados.

Nótese que, hasta ahora, no hay nivel 1 para este proceso, porque no hemos podido elaborar ítems con una demanda menor a la del nivel 2. Quizás se encuentre que esto es posible en la producción futura de ítems.

Combinar las descripciones para cada nivel con los procesos genera la escala general para la alfabetización científica. Las tareas en el nivel 1 requieren que los estudiantes:

a) recuerden simple conocimiento científico factual;

b) usen conocimiento común de la ciencia para sacar o evaluar conclusiones.

Lo que un estudiante necesita ser capaz de hacer para completar una tarea en el nivel 5 es:

a) crear o usar modelos conceptuales sencillos para hacer predicciones o dar explicaciones;

\section{AGRADECIMIENTO}

Este trabajo presenta un panorama de los resultados de un trabajo de grupo de los miembros del SEG de ciencias, asistidos por Steven Bakker e Ico deRoo de CITO y por Jan Lokan de ACER.

\section{NOTAS}

- Ponencia presentada en el VICongreso Internacional sobre Investigación en la Didáctica de las Ciencias (Barcelona, 12 al 15 de septiembre de 2001). Ha sido traducida del inglés por Agustín Adúriz-Bravo.

${ }^{1}$ Este trabajo recurre a material publicado en Harlen, W. (2001). The assessment of scientific literacy in the OECD/PISA project. Studies in Science Education, 36, pp. 79-104.

\section{REFERENCIAS BIBLIOGRÁFICAS}

ANGELL, C., KJAERNSLI, M. y LIE, S. (2000). Exploring students' responses on free response science items in TIMSS, en ShorrocksTaylor, D. y Jenkins, E.W. (eds.). Learning from others: International comparisons in education, pp. 159-188. Dordrecht: Kluwer.

HARLEN, W. (2001). The assessment of scientific literacy in the OECD/PISA project. Studies in Science Education, 36, pp. 79-104.

FENSHAM, P.J. (1985). Science for all. Journal of Curriculum Studies, 17, pp. $415-435$. b) analizar investigaciones científicas en relación con, por ejemplo, el diseño experimental, la identificación de las ideas que se están poniendo a prueba;

c) relacionar datos como pruebas para evaluar puntos de vista alternativos o diferentes perspectivas;

d) comunicar argumentaciones científicas o descripciones en detalle y con precisión.

\section{COMENTARIO CONCLUSIVO}

El PISA comparte con otras encuestas a gran escala el destino de ser un compromiso entre lo ideal y lo posible. Sin embargo, representa un avance respecto de anteriores encuestas internacionales en ciencias al enfocarse sobre lo que los estudiantes podrían tomar de sus estudios para abordar los elementos científicos de la vida cotidiana. Por supuesto, no podemos suponer que los estudiantes llegan a las respuestas que dan a través de los procesos que los ítems de prueba parecen requerir; tampoco que, si así sucede, esto es resultado de su educación científica. Se necesitan otros estudios asociados (como el que condujeron Fensham y Haslam (1998) en relación con las interpretaciones que hacen los estudiantes de los ítems del TIMSS) a fin de investigar no sólo lo que los estudiantes creen que se pregunta en los ítems y los procesos a través de los cuales llegan a sus respuestas, sino también de dónde sacan la comprensión relevante. Por ejemplo, ¿usan información obtenida de fuentes no escolares, de internet, de los medios de comunicación, de los museos de ciencias? Las respuestas probablemente variarán de país en país, de acuerdo con las oportunidades de educación informal disponibles, pero, a menos que se investigue más sobre esta cuestión, se corre el riesgo de sacar conclusiones inapropiadas acerca de los currículos escolares.

${ }^{2}$ Los países de la OECD que participan son: Australia, Austria, Bélgica, Canadá, República Checa, Dinamarca, Finlandia, Francia, Alemania, Grecia, Hungría, Islandia, Irlanda, Italia, Japón, Corea, Luxemburgo, México, los Países Bajos, Nueva Zelanda, Noruega, Polonia, Portugal, España, Suecia, Suiza, el Reino Unido, los Estados Unidos. Los países no miembros de la OECD que participan en 2000 son: Brazil, Letonia, la Federación Rusa y China.

${ }^{3}$ Los miembros del SEG de ciencias son: Wynne Harlen (coordinadora), Peter Fensham, Raul Gagliardi, Donghee Shin, Svein Lie, Manfred Prenzel, Senta Raizen, Elizabeth Stage.

${ }^{4}$ www.pisa.oecd.org.

FENSHAM, P.J. y HASLAM, F. (1998). Conceptions of context in extended response items in TIMSS. Trabajo presentado en el Congreso de la ASERA, Darwin, julio de 1998.

LAUGKSCH, R.C. (2000). Scientific literacy. Science Education, 84(1), pp. 71-94.

OECD (1999). Measuring student knowledge and skills. A new framework for assessment. París: OECD.

OECD (2000). Measuring student knowledge and skills: The PISA assessment of reading, mathematical and scientific literacy. París: OECD. 